\title{
On the Cultivation of Students Self-Regulated Learning Ability in Chinese Teaching
}

\author{
Hanyuan Yang* \\ Zhuodaoquan Primary School, Wuhan, Hubei Province, China \\ *Corresponding author. Email: yhyuan_68@163.com

\begin{abstract}
It's the important moment for students in the primary school stage to consolidate the basic knowledge. Forming good study habits is helpful to lay the foundation for students' future study career. With the continuous development of all aspects of our society, more and more attention has been paid to students' education. With further requirements for curriculum revolution in new curriculum reform, schools all over the country actively responded and improved current teaching methods in order to develop students' independent learning ability and improve their learning interests. In view of the present situation of primary school Chinese classroom education, this paper studies the factors that influence the cultivation of students' independent learning ability in primary school Chinese teaching, and further puts forward the optimization measures to cultivate students' independent learning ability according to the influencing factors, so as to effectively improve students' enthusiasm for learning and promote their all-round development.
\end{abstract}

Keywords: Primary school Chinese, Self-regulated learning ability, Cultivation.

\section{浅析语文教学中学生自主学习能力的培养 杨汉元 ${ }^{*}$}

卓刀泉小学, 湖北武汉, 中国

“通讯作者. 邮箱: yhyuan_68@163.com

\section{中文摘要}

学生在小学阶段是巩固基础知识的重要阶段, 养成良好的学习习惯, 有助于为学生今后的学习奠定基础。随着 我国社会各方面的不断发展, 对学生教育的重视程度也越发严格。自新课改提出对课程改革的进一步要求, 各 地学校积极响应, 为培养学生自主学习能力, 提高学生学习兴趣改进当前的教学方法。本文针对当下小学语文 课堂教育的现状, 研究影响小学语文教学自主学习能力培养的因素, 并根据影响因素进一步提出培养学生自主 学习能力的优化举措, 从而有效提高学生的学习热情, 促进学生的全方面发展。

关键词: 小学语文; 自主学习能力; 培养

\section{1. 引言}

在当今新时代的大背景下，国家政府越发地注重 学生教育, 而学生作为国家未来的建设者和接班人身 上也肩负着重要的使命。基于现行的新课改要求以及
推动学生综合素质教育之下, 培育学生自主学习能力 成为当前小学语文教学中的首要任务。而受到传统教 育观念的影响, 当前小学语文课堂教学中仍存在着诸 多不足, 除了需要针对教师的教学方法和教学观念进 行改进, 还需要进一步地提高学生的学习兴趣和学习 
热情, 培养落实学生自主学习能力。

\section{2. 当前小学语文教学现状分析}

语文学科作为我国教育中的重要构成, 因此对小 学语文教育方法的改革也受到了重视。首先由于语文 是我国母语, 学生都有一定的语言基础, 对语文课堂 所讲述的知识也有一定的接受能力。但受到我国传统 教育的影响, 在语文教育过程中往往只重视学生的成 绩, 而忽视了学生的学习方法, 判别学生成绩的标准 只有一张试卷。尤其是在小学阶段, 正是学生打牢基 础的时段, 不当的教学方法会使学生养成不良的学习 方法, 从而大大降低学生学习兴趣以及学习能力。在 当前的语文教学中, 教师主要针对语言文字、文字理 解、语言表述, 写作技巧等方面进行辅导。在这一前 提下大大忽视了学生能力养成, 使其在学习过程中只 注重背诵书本的知识, 轻视了对学习方法的运用, 不 利于学生养成自主学习能力, 也不利于其今后的学习 和生活。

\section{3. 语文教学小学生自主学习的必要性}

在教学过程中由于学生与学生之间的差异, 往往 会存在教学方法无法包容整体学生的情况。随着时代 的不断进步, 为进一步满足学生差异化发展, 为提高 学生学习主动意识有了新的要求。在学生自主投入到 学习的过程中, 往往会引起自身对学习的兴趣, 由兴 趣带动学习, 从而进一步激发学生潜能。

随着信息化时代的飞速发展, 当前世界知识储备 已经达到一个新的高度。学生在学校所能接受的教育 是局限的, 因此学生若想进一步充实自身头脑知识, 首先要做到的便是培养自身自主学习能力。通过自主 意识主动投入到学习中去, 将书本理论知识进一步地 与现实实际相结合, 而获得更多的发展机会。

\section{4. 影响语文自主学习的主要因素}

首先学生在语文学习过程中未能形成明确的学习 目标, 使得学习方向出现偏差, 往往会存在不重视语 文学习的情况。学生缺乏自主学习动力, 未能意识到 语文学习对今后发展的重要性, 使得其在学习时往往 抱有侥幸心理, 利用原本的语文知识储备搪塞相关的 语文考试。

好的习惯的养成决定了学生未来的发展。在学生 的学习过程中, 首先受到学生对语文学习兴趣影响, 其次缺乏良好的学习习惯进一步的阻碍了当前的语 文教育。就目前的语文课堂现状而言, 大部分学生欠 缺正确的学习方法, 未能养成良好的学习习惯, 对语 文学习的投入得不到相应的回报, 渐渐失去对语文学 习的兴趣, 从而导致语文成绩逐渐下降。

\section{5. 语文教学培养小学生自主学习能力的优 化举措}

\section{1. 提高学生学习兴趣, 培养学生养成良好}

\section{的学习习惯}

教师在教学过程中应注重培养学生的学习兴趣, 将语文教学变得更加具备趣味性。在教学过程中通过 引入有趣的活动、或者相关的语文知识科普, 使语文 教学更加贴近学生实际生活, 激发学生的进取心和求 知欲。学生在学习的过程中缺乏对所学知识的总结能 力, 不懂得主动研究学习方式方法。因此教师在做到 教育改革的同时, 应该以解决学生学习方法问题为中 心, 带动学生自主学习, 使其掌握学习的方法, 从而 让今后的学习生涯事半功倍。

\section{2. 通过问题引导学生建立活跃的思维方}

\section{式}

随着时代的发展进步, 语文教学已经不能局限于 传统的教学模式, 在教学中通过问题引导学生独立思 考, 可以进一步地促进学生养成正确的思维模式, 从 而培育其自主学习能力。在教学过程中教师主要起到 辅导作用, 当学生遇到问题时应给予对方一定的思考 和解决时间, 使学生提出质疑, 在疑惑中逐渐掌握解 决问题的方法, 以及培养其独立思考解决问题的能力。 在传统的语文教学中使用灌输式教学方法, 往往呈现 的是教师讲课、学生听课的固态思维, 在此过程小学 生属于被动接受知识的状态, 甚至在某些特定时间, 例如上课走思、开小差等时间段, 对知识的接受程度 几乎为零, 由此大大降低了教师的授课效率。而利用 问题引导式教学方法, 教师在其中只扮演引领者的角 色, 通过调动学生的积极性, 使学生发散思维提高自 身创造意识, 培养自身自主学习能力, 在今后的学习 生涯中遇到问题可以积极主动解决。

\section{3. 革新教学观念, 创新教学方法}

在传统的教学理念中, 学生的检测成绩往往是教 师对教学成果的评估标准, 过于重视学生的分数, 从 而忽视了学生在学科上的综合教育。随着时代的改变, 传统教学方式已经不适应于当下的课堂需求, 无法满 足学生的语文学习。若想真正的培养学生自主学习能 力, 培育具备综合素质的新型人才, 作为教师首先应 该转变固有思想, 创新教学方法, 对教学理念不断更 新完善。除此之外, 语文教师可以针对教学实际开展 实践教学模式, 由于语文学科的特殊性, 其实践活动 可以进一步结合语文知识, 开发学生思维, 促进学生 之间的团结合作, 使学生在实践过程中养成良好的自 主学习能力。 


\section{4. 培养学生的自主阅读能力, 提升学生的}

\section{知识储备}

培养学生的自主阅读能力, 首先应该提高学生的 阅读兴趣, 养成良好的阅读习惯。为此, 教师可以在 班级中打造书香图书角, 为学生提供充足的课外读物, 并且教师自身起到榜样和带动作用, 陪同学生一同积 极投入到阅读中去, 提高学生在阅读中的满足感。除 此之外, 培育学生养成在学业过程中主动收集资料的 习惯，有效提高学生的阅读效率。除了对阅读量以及 阅读兴趣的重视外, 也应该进一步培育学生对阅读内 容的质疑能力。促使学生在阅读过程中, 主动去发掘 书本中的难点, 通过发现问题解决问题的过程, 进而 培养学生的自主思考能力, 在思考中融入创新观点, 提高自身创造性。

\section{5. 给予学生学习自主权}

教师在语文教学的过程中, 除了需要进一步对学 生各方面做到全面了解, 保证学生教育的全面覆盖, 还要进一步做到对学生的解放。使学生在传统的教学 方式中走出来, 学习不再只是局限于课本知识, 对于 其他课外知识的培育也至关重要。解放学生的思维方 式, 增加学生在思考过程中的创造性, 给予学生发表 自身感受的权利, 使学生感受到学习主动权的魅力。 在自主学习的过程中, 往往可以进一步建设学生自信 心, 使学生具有主动性, 有效挖掘学生学习潜力, 有 效提高学生学习效率。

在学习过程中, 受到我国传统思想的影响, 学生 往往秉承的尊师敬长的理念与教师进行交往。在此情 况下, 为进一步拉近师生之间关系, 提高语文教学的 效率, 需要教师主动去打破这层隔阂, 在教学过程中 积极鼓励学生去表达自己内心看法, 以及在学习过程 中遇到的问题。通过拉近与学生之间的关系, 使师生 之间的相处模式更加平等, 从而有效帮助教师走进学 生内心, 发现学生闪光点, 进一步用学生最容易接受 的方式答疑解惑, 以此来提高语文课堂教学质量。

\section{6. 结语}

语文是渗透进我们生活中的一门学科, 在我们生 活的方方面面都发挥了至关重要的作用。因此对学生 语文知识和思维的培养, 是当代老师首要把握的中心 思想。随着教学方式在新课改的要求下不断完善, 教 师本身也应该转变教学思维, 在教导学生基础知识之 余, 增强学生自主学习能力, 通过多方面入手发现学 生自身闪光点, 从而进一步培养学生综合素质。在教 学过程中, 教师应该注重与时俱进, 对自身教学观念 不断创新, 结合学生个性因材施教, 有效促进学生的 全面发展。

\section{REFERENCES}

[1] Xiaoxia Wang. How to improve students' autonomous learning ability in primary school Chinese teaching [J]. China Rural Education, 2020(13):51-52.

[2] Yunyun Huang. Research on the Cultivation of Students' Autonomous Learning Ability in Chinese Classroom Teaching in Primary Schools [J]. Theoretical Research and Practice of Innovation and Entrepreneurship, 2020, 3(07): 32-33.

[3] Yuhong Wang. How to Cultivate Students Autonomous Learning Ability in Primary School Chinese Teaching [J]. Quality Education in Western China, 2020, 6(06): 58-60.

[4] Xicheng Zhong. On the Cultivation of Students' Autonomous Learning Ability in Chinese Teaching in Primary Schools [J]. Huaxia Teachers, 2019(33): 18.

[5] Chunling Liu. Exploring Cultivating Students' Self-directed Learning Ability in Primary School Chinese Classroom [J]. China Rural Education, 2019(32): 106. 